\title{
CORRECTION
}

\section{Correction: EphB2 in the medial prefrontal cortex regulates vulnerability to stress}

Ruo-Xi Zhang ${ }^{1,2,3}$, Ying Han ${ }^{1,2,3}$, Chen Chen ${ }^{1}$, Ling-Zhi Xu ${ }^{2,3}$, Jia-Li Li ${ }^{2}$, Na Chen ${ }^{2}$, Cheng-Yu Sun ${ }^{1,2}$, Wen-Hao Chen ${ }^{1}$, Wei-Li Zhu ${ }^{2}$, Jie $\mathrm{Shi}^{2}$ and $\mathrm{Lin} \mathrm{Lu}^{1,2,4}$

Neuropsychopharmacology (2019) 44:1339-1343; https://doi.org/10.1038/s41386-019-0364-1

Correction to: Neuropsychopharmacology https://doi.org/10.1038/ npp.2016.58, published online 22 April 2016.
Following publication of the above article, the authors noticed that an incorrect version of Figs. $2 \mathrm{f}, 3 \mathrm{f}, 5 \mathrm{~h}$ and $7 \mathrm{~d}$ was presented.

The corrected Figures appear below, and the corrected Supplementary Information file is appended.

\footnotetext{
${ }^{1}$ Institute of Mental Health, National Clinical Research Center for Mental Disorders, Key Laboratory of Mental Health and Peking University Sixth Hospital, Peking University, Beijing, China; ${ }^{2}$ National Institute on Drug Dependence and Beijing Key Laboratory of Drug Dependence, Peking University, Beijing, China; ${ }^{3}$ Department of Pharmacology, School

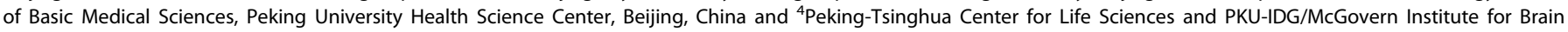
Research, Peking University, Beijing, China

Correspondence: Lin Lu (linlu@bjmu.edu.cn)

These authors contributed equally: Ruo-Xi Zhang, Ying Han, Chen Chen
}

Published online: 19 March 2019 
a Timeline

\begin{tabular}{|c|c|c|c|}
\hline $\begin{array}{c}\text { Chronic social } \\
\text { defeat stress }\end{array}$ & 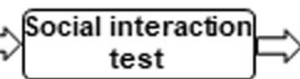 & $\begin{array}{c}\text { Behavioral tests } \\
\text { (SI, SPT, NSFT) }\end{array}$ & $\Rightarrow \begin{array}{c}\begin{array}{c}\text { Quantification } \\
\text { analysis of protein }\end{array} \\
\text { aly }\end{array}$ \\
\hline D1 - D10 & D11 & D12 - D14 & D15 \\
\hline
\end{tabular}

b Social interaction test
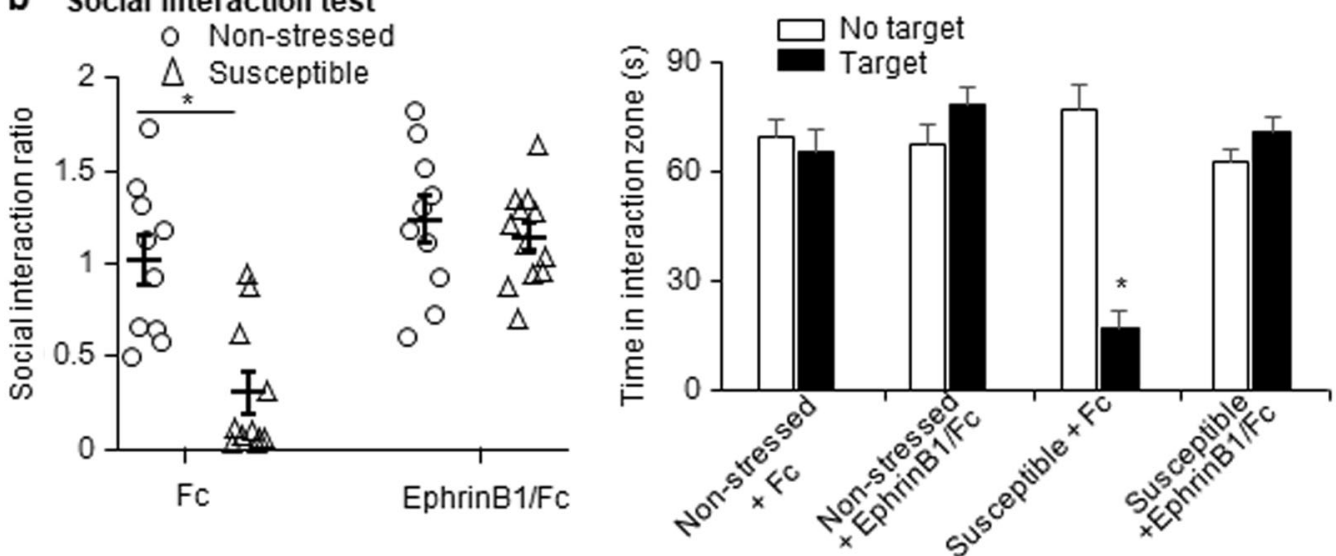

c

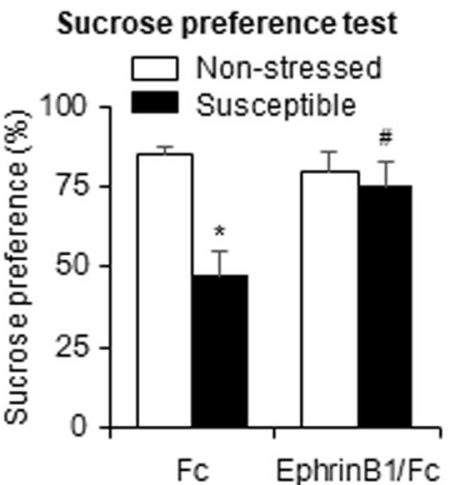

d

Novelty suppressed feeding test
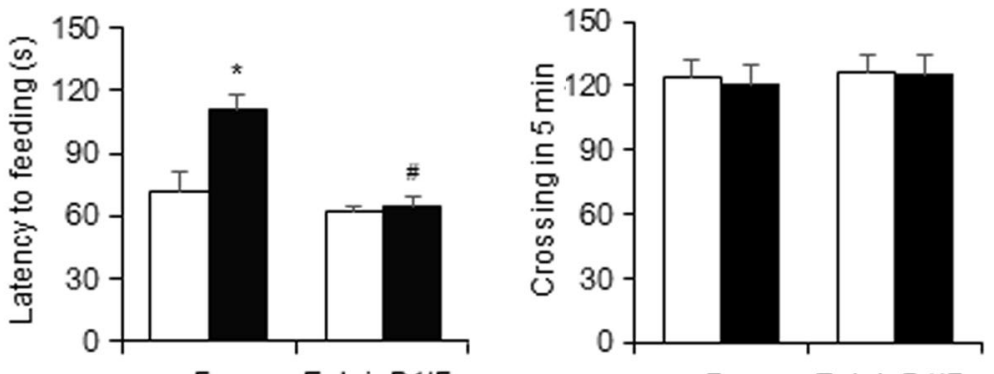

$f$ Protein levels in mPFC
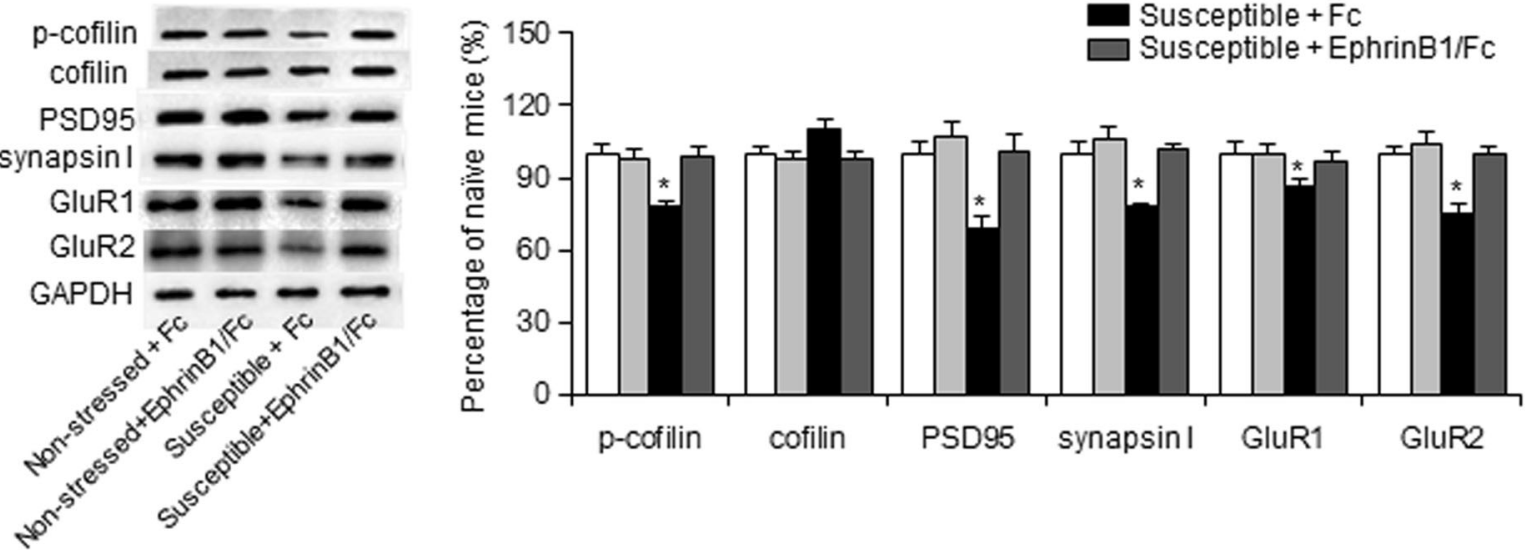

Fig. 2 
a Timeline

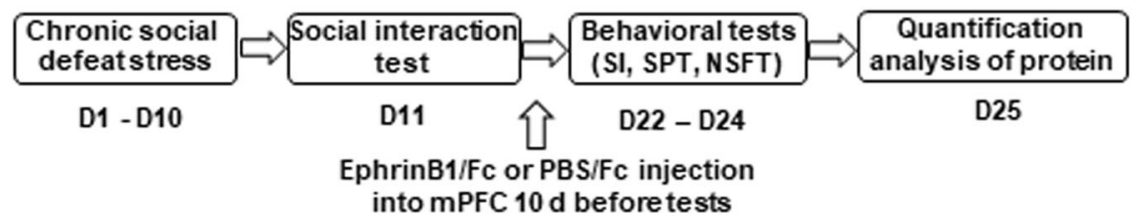

b Social interaction test
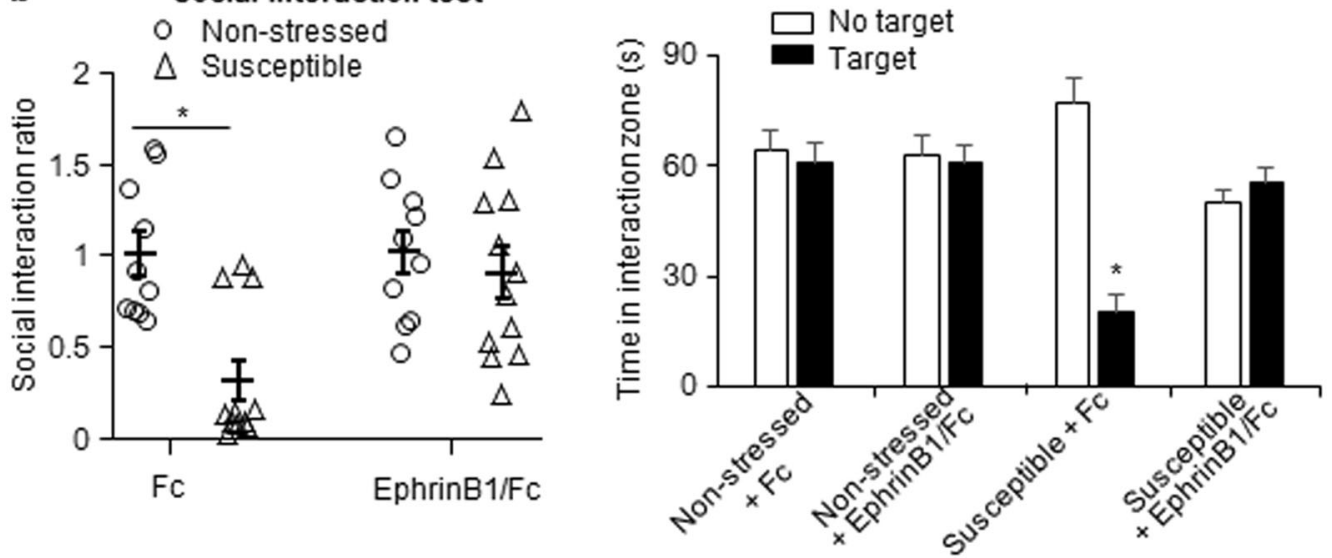

C Sucrose preference test

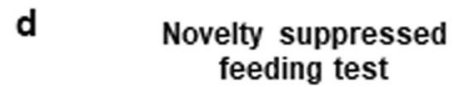

e Locomotor activity
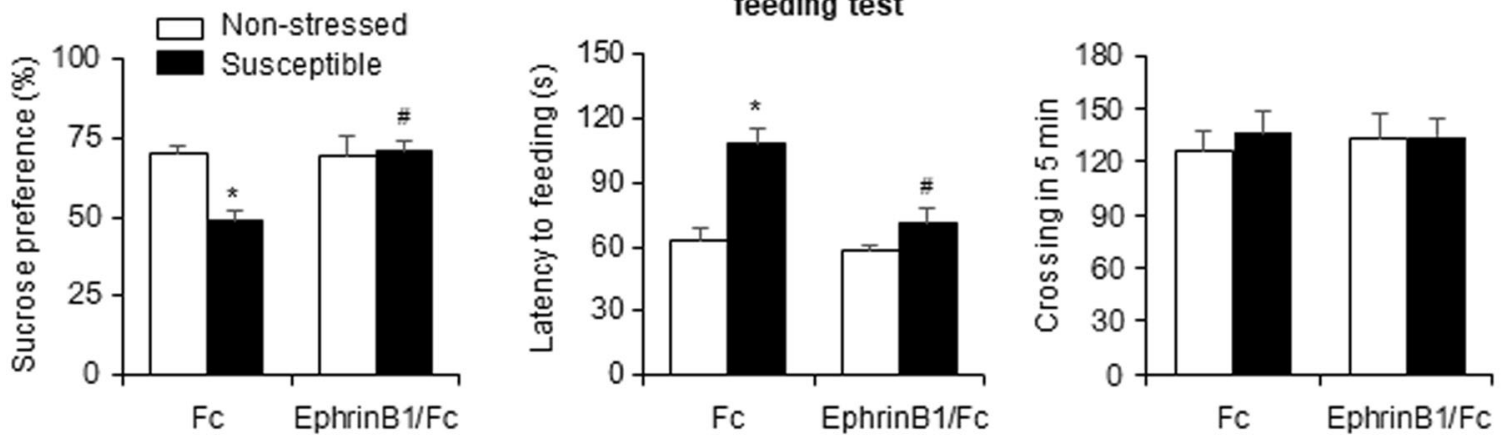

f Protein levels in MPFC
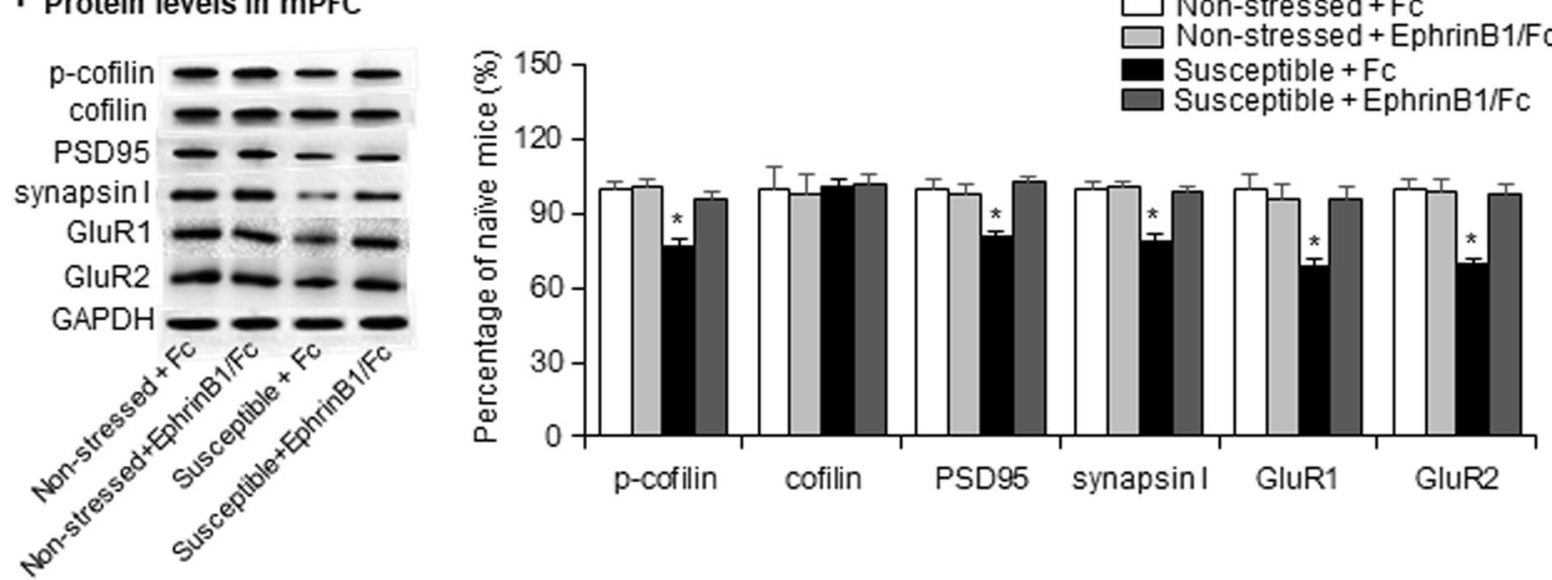

Fig. 3 
a

Timeline

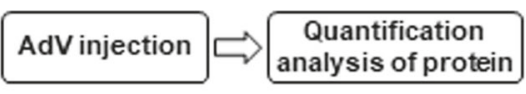

D1

D8

b

Representative pictures
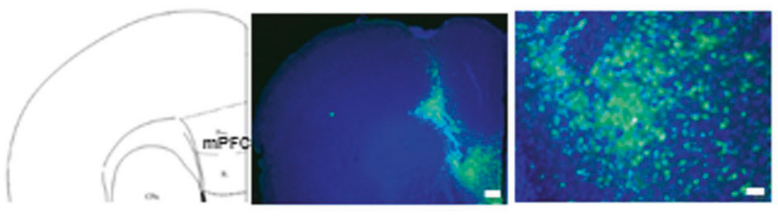

c

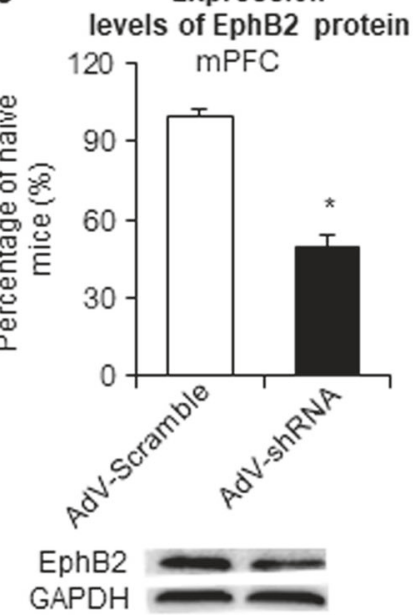

d Timeline

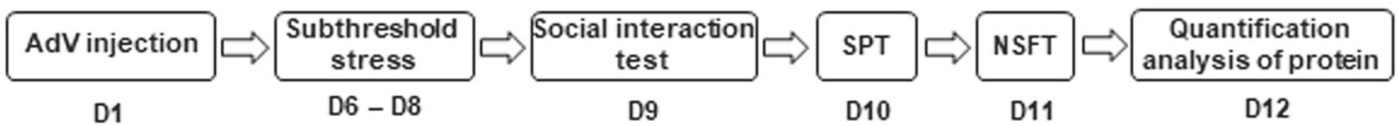

e Social interaction test

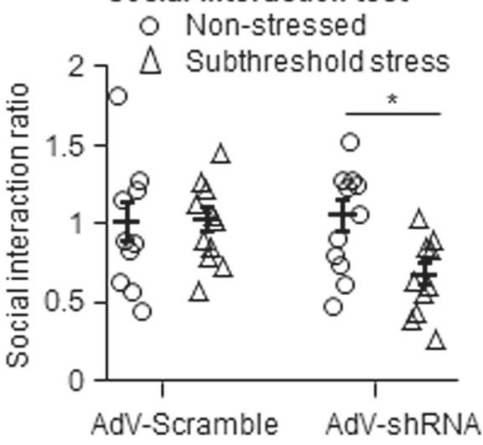

f Sucrose preference test

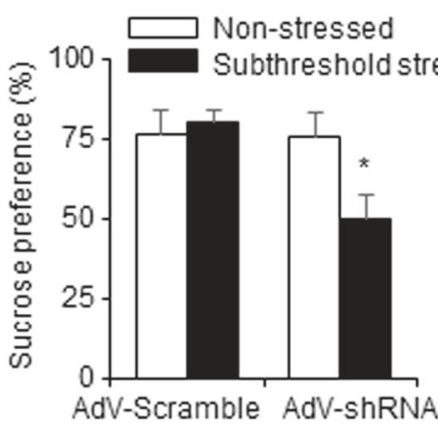

Novelty suppressed feeding test

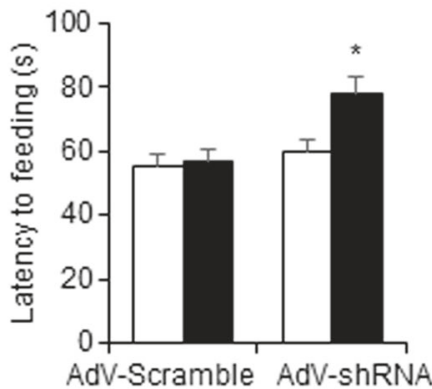

h Protein levels in MPFC
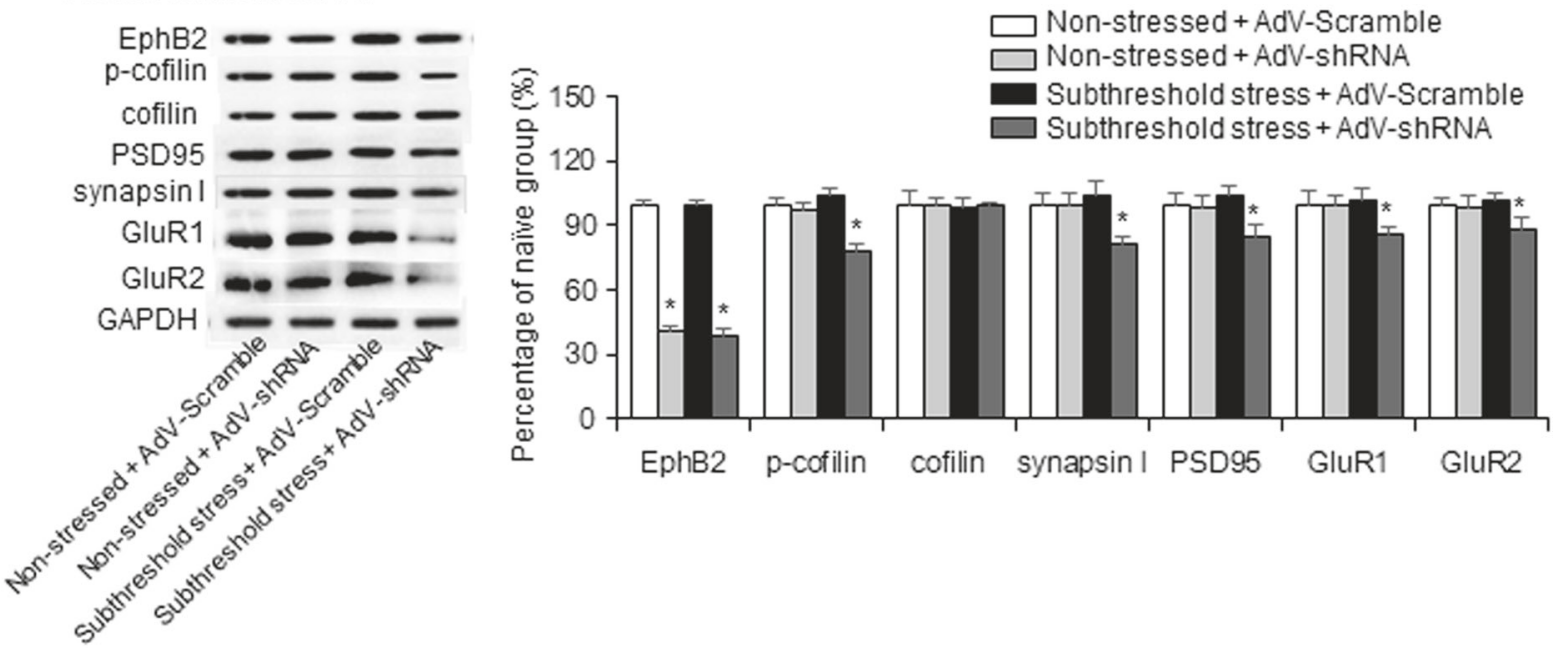

Fig. 5 
a Timeline

\begin{tabular}{|c|c|c|c|c|}
\hline $\begin{array}{c}\text { Chronic social } \\
\text { defeatstress }\end{array}$ & 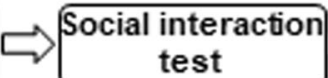 & $\begin{array}{l}\text { EphrinB1/Fc or } \\
\text { PBS/Fc injection }\end{array}$ & 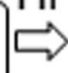 & Perfusion \\
\hline
\end{tabular}

D1 - D10

D11

D12

b Spine numbers

Non-stressed + Fc

Non-stressed + EphrinB1/Fc

Susceptible + Fc

Susceptible + EphrinB1/Fc

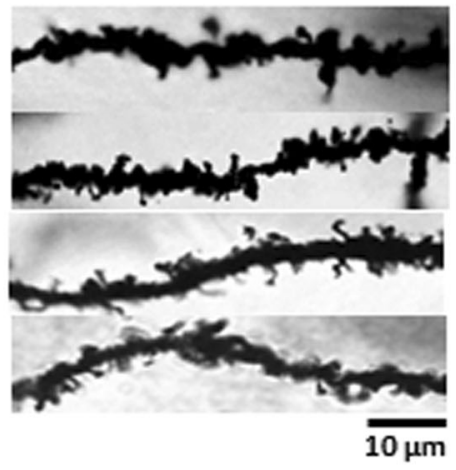

C Timeline

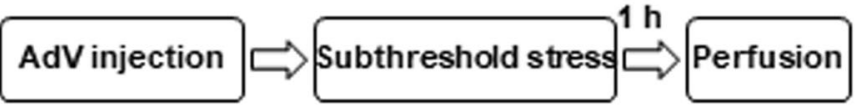

D1

D6 - D8

\section{d Spine numbers}

Non-stressed + AdV-Scramble

Non-stressed + AdV-shRNA
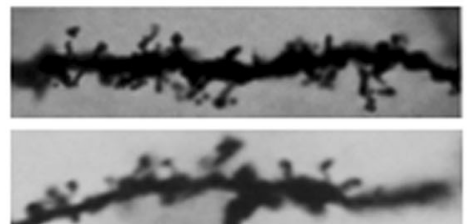

Subthreshold stress + AdV-Scramble

\section{(1)}
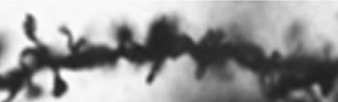

Subthreshold stress + AdV-shRNA

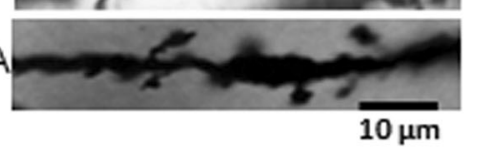

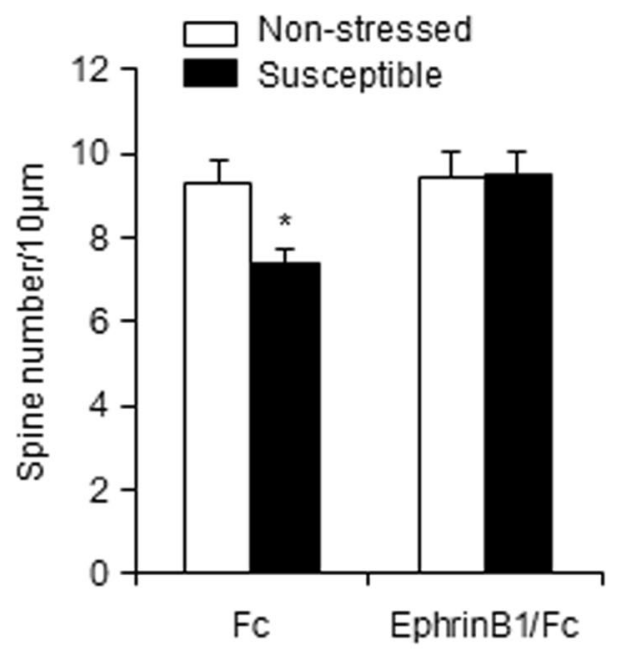

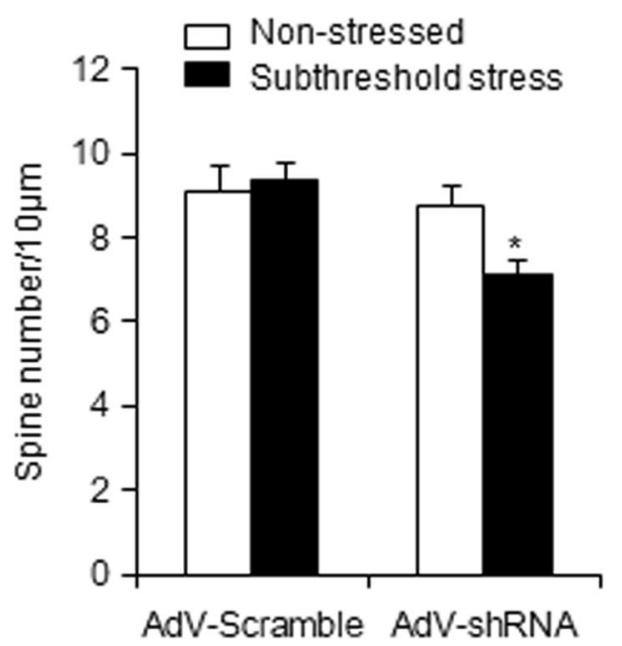

Fig. 7 